\title{
Circadian rhythms of angiogenic factors in skin and wound tissue in per 2-mutant mice
}

\author{
Tomoaki Hoshi ${ }^{1}$, Yoko Toukairin ${ }^{1}$, Tomomi Arai ${ }^{1}$ and Makoto Nogami ${ }^{1 *}$ \\ ${ }^{1}$ Department of Legal Medicine, Teikyo University School of Medicine, Tokyo, Japan
}

\begin{abstract}
It has recently been reported that circadian gene Period 2 (per2) regulates mRNA expression of vascular endothelial growth factor gene (Vegf) in some tumor cells. It still remains unknown, however, whether per 2 has an effect on expression of angiogenic factors during the wound healing process. To investigate the circadian rhythms of angiogenic factors in intact skin and wound tissue in per2-mutant and control mice, we used a skin incision wound model on per2-mutant and control mice, and analyzed circadian rhythms of mRNA levels for several angiogenic factors. Our results show a novel finding that, for some angiogenic factors, the circadian rhythms of mRNA levels in wound tissue exist in per2-mutant as well as in control mice, indicating that some circadian rhythms may be per2-independent. We observed circadian rhythms of per2 and some angiogenic factor mRNAs in control mouse intact skin. The circadian patterns of some angiogenic factors mRNAs in intact skin were altered in per2-mutant mice relative to normal mice, indicating the existence of per2-dependent and independent regulations of angiogenic factors expressions.
\end{abstract}

\section{Introduction}

The evaluation of wound healing stage is important in forensic pathology. Our research has previously focused on angiogenesis and lymphatic regeneration in skin incision wounds $[1,2]$. We showed that vascular endothelial growth factor-A (VEGF), an important angiogenic factor, is produced in the rat skin incision wound area from an early stage. We further showed that VEGF is immunohistochemically localized in leucocytes [1] and mainly in the wound area [3].

In a study focusing on the molecular mechanism of VEGF, Koyanagi et al. showed that the levels of VEGF mRNA in tumor cells implanted in mice substantially increase in response to hypoxia, and interestingly, the levels fluctuate rhythmically in a circadian fashion [4]. Circadian gene Period 2 (per2) and another circadian gene, Cryptochrome1, whose expression in the implanted tumor cells exhibits a circadian oscillation, inhibit the hypoxia-induced Vegf promoter activity [4]. Other studies demonstrated that Period 1 (per1), another member of the Period family, and per 2 exhibit circadian oscillations in mRNA expression in human peripheral leucocytes [5-8].

Per2-mutant mice have been shown to be more resistant to ischemic myocardial injuries, presumably by interfering with another circadian gene product Clock-Bmall, whose activity represses transcription targets, including VEGF [9]. Therefore, it is conceivable that VEGF mRNA expression by neutrophils infiltrating in the wound tissue may be under the influence of circadian gene per 2 oscillation. On the other hand, per2-mutant mice develop larger infarct sizes by myocardial ischemia compared with wild-type mice, and lose the cardioprotection conferred by ischemic preconditioning [10]. This impairment is most likely caused by a failure to stabilize hypoxia-inducible factor1alpha (HIF1a) [10]. Per2 suppresses Clock-Bmal1 and Clock-Bmal2dependent transactivation of the plasminogen activator inhibitor 1 gene (Pai1/ Serpine1) promoter in vitro [11]. Therefore, the role of per2 in ischemic injury is yet to be determined.

To our knowledge, the possible role for per 2 in angiogenesis during the wound healing process has not been explored yet. In this study, first we report on the circadian rhythms of per2 and angiogenic factor mRNAs in the normal skin. We then hypothesized that angiogenic factors produced by leucocytes and other infiltrating cells in the wound area are under the influence of per2. To test this hypothesis, we applied the incision wound model to per2-mutant and control mice to examine the mRNA expression levels of angiogenic factors: VEGF-A (VEGF), hepatocyte growth factor (HGF), HIF1a, fibroblast growth factor 1 (FGF1), angiopoietin 1 and 2 (ANGPT1 and 2), angiopoietinlike proteins 1 and 2 (ANGPTL1 and 2), PAI1, granulocyte colony stimulating factor (CSF3/G-CSF), chemokine (C-X-C motif) ligand2 (CXCL2), matrix metallopeptidase 9 (MMP9), transforming growth factor beta 1 (TGFb1), colony-stimulating factor 2 (CSF2), and antiangiogenic factor tenomodulin (TNMD) $[12,13]$.

We also performed histological analysis of tissue collected 7 days after the skin incision, to evaluate the endothelial cells in the wound healing process in per2-mutant and control mice to examine the effect of per2 clock gene on angiogenesis [1,2].

\section{Materials and methods}

\section{Animals}

Six-week-old male B6.Cg-Per2tm1Brd Tyrc-Brd/J mice (per2mutant mice) and $\mathrm{B} 6(\mathrm{Cg})-\mathrm{Tyrc}-2 \mathrm{~J} / \mathrm{J}$ mice (control mice) were purchased from the Jackson Laboratory (Bar Harbor, Maine, USA), and maintained in our institution [12]. The mutant mice harbors a deletion mutation in the PAS domain (a dimerization domain found in Per, Arnt and Sim) [12]. The mice were exposed to luminescent light during the daytime (8 A.M. through 8 P.M.), and kept in the dark at

Correspondence to: Makoto Nogami, Department of Legal Medicine, Teikyo University School of Medicine, 2-11-1, Kaga, Itabashi-ku, Tokyo, 173-8605, Japan, Tel: +81 33964 1211; E-mail: mnogami@med.teikyo-u.ac.jp

Key words: circadian rhythm, per2, angiogenesis, mouse, wound

Received: March 05, 2017; Accepted: April 11, 2017; Published: April 14, 2017 
night. The approximate intensity of the light in the daytime was 600 lux. For ZT18 experiments, the daytime was shifted to 8 P.M. through 8 A.M. The experiments were approved by the animal ethics committee of the institution (Approval No.12-043).

\section{Skin incision}

We have previously reported the mRNA expression levels of various angiogenic factors found in wound tissues at 24 hours after a skin incision [13]. Therefore, for this study we also chose the 24 hour as a time point after the skin incision to measure the mRNA expression levels in tissues at various Zeitegeber time (ZT) points [13]. Of note, ZT is a 12 hour light-12 hour dark cycle, with ZT0 representing the time when lights are turned on and ZT12 the time when lights are off. (Figure 1)

Mice were anesthetized by isoflurane inhalation, and 5 centimeters of the dorsal skin was incised to the depth of the bottom of the subcutaneous tissue using a sterile scalpel and scissors at ZT2, ZT6, ZT11, and ZT18 time points. Twenty-four hours after the skin incision, the incised skin lesion and the intact skin area were collected under isoflurane inhalation for mRNA and histological studies (Figure 1).

For CD31 immunohistochemical study, the skin wound tissues were made at ZT2, and collected 7 days after the skin incision [1,2].

\section{Semi-quantification of angiogenic factor mRNAs by real time RT-PCR}

Total RNA was isolated from the intact skin and the dermal skin wound tissue using QIAMP RNeasy fibrous tissue kit (QIAGEN, Tokyo). The relative expressions of various angiogenic and lymphagiogenetic factors were determined by TaqMan Gene Expression Assay and TaqMan Fast Virus 1-Step Master Mix (Fischer Scientific, USA), using the StepOne Plus (Fischer Scientific, USA) real-time PCR system [13]. The factors studied were as follows with TaqMan Gene Expression Assay primer IDs in parentheses : VEGF (Mm01281449_m1), HGF (Mm01135193_m1), TGFb1 (Mm01178820_m1),HIF1a(Mm00468869_m1), FGF1(Mm00438906_ m1), ANGPT1 (Mm00456503_m1), ANGPT2 (Mm00545822_m1), ANGPTL1 (Mm01291815_m1), ANGPTL2 (Mm00507897_m1), PAI1 (Mm00435860_m1), CSF2 (Mm01290062_m1), CSF3 (Mm00438335_ g1), CXCL2 (Mm00436450_m1), MMP9 (Mm00442991_m1), and TNMD (Mm00491594_m1).

For the circadian rhythm evaluation, RNA from intact skin of per2-control mice at ZT2 was pooled and used as the calibrator. The delta CT was calculated using actin mRNA as the reference gene, and the difference of delta CT (delta-delta CT) was calculated between the intact skin at various ZTs and the calibrator. The data were expressed as the relative quantity (2 to minus delta-delta CT) [14].

For the wound mRNA evaluation, the delta CT was calculated using actin mRNA as the reference gene, and the difference between the wound and the control intact skin tissue delta CT (delta-delta CT) was calculated. The data were expressed as the relative quantity fold changes ( 2 to minus delta-delta CT) [14].

\section{Histological study and immunohistochemistry of capillaries in the wound areas}

The wound tissues were fixed in $4 \%$ paraformaldehyde for 3 $\mathrm{hr}$, embedded in paraffin, and 3 micrometer paraffin sections were prepared. The numbers of leucocytes in the wound areas were counted on samples stained with hematoxylin and eosin (HE) on pictures using ImageJ software (http://imagej.nih.gov), and the results expressed as leucocytes per square millimeter.
Immunohistochemistry for endothelial marker CD31 was performed using Histofine simple stain mouse MAX-PO (Rat) (Nichirei \#414311, Japan). Briefly, 6 micrometer-thick sections were dewaxed in xylene, and in $10 \mathrm{mM}$ citrate buffer (pH6.0) in autoclave at $121^{\circ} \mathrm{C}$ for $10 \mathrm{~min}$. Then, sections were pretreated in $3 \%$ hydroxyperoxide, at room temperature for $10 \mathrm{~min}$, washed in $0.05 \mathrm{M}$ phosphate-buffered saline (PBS, pH7.6) 3 times, and blocked with Blocking I (Nakaraitesk \#03953-95, Japan) for $10 \mathrm{~min}$. The sections were incubated with the rat monoclonal anti-mouse CD31 (PECAM-1) antibody, Clone SZ31 (Dianova \#DIA-310, Japan) diluted 20 to 100-fold in DAKO Antibody Diluent with Background Reducing Components (DAKO, Japan) overnight at $4^{\circ} \mathrm{C}$. After washing in PBS, the sections were incubated with Histofine simple stain mouse MAX-PO (Rat) (Nichirei, Japan) at room temperature for $30 \mathrm{~min}$. After washing with $\mathrm{PBS}$, the sections were stained with diaminobenzidinetetrahydrochloride and hydroperoxide. The percentages of the vascular areas to the total areas were calculated on pictures using ImageJ software. The sizes from 50 pixels and above were counted on the software.

\section{Statistical analysis}

Data were expressed as means \pm SEM. To exclude outlying data, GraphPad Prism software ver.6 and 7 (La Jolla, CA, USA) was used. Then, Kruskal-Wallis non-parametric test was used for data evaluation. A p-value smaller than 0.05 was considered statistically significant.

\section{Results}

\section{Per2 and angiogenic factors' mRNA levels in mouse skin oscillate during the day}

We investigated whether or not per2 plays a role in tissue healing after injury. First, we analyzed the per 2 mRNA levels on intact mouse skin. In accordance with the previous report [15], depending on the time of day, per2 mRNA levels oscillated (Figure 2). The highest level of per2 mRNA was detected at ZT11 and the lowest level at ZT2, just at the beginning of the light condition. Between the highest and lowest points, the levels steeply declined and increased.

The mRNA levels of angiogenic factors in control mouse intact skin displayed circadian rhythmicity, as well (Figure 3). The mRNA levels of different factors exhibited distinct patterns of circadian rhythm in intact skin of control mice. Interestingly, the results of ANGPTL1, ANGPTL2, CSF2, MMP9, and TNMD's mRNA levels mirrored the per2 mRNA pattern; the highest relative levels were detected at ZT11, right before the beginning of the dark period. Other angiogenic factors showed different patterns (Figure 3).

We then compared circadian rhythms of angiogenic factor mRNAs between per2-mutant and control mice (Figure 4). Among the angiogenic factors with ZT11 peaks, ANGPTL1 and CSF2 mRNAs lost peaks at ZT11 in per2-mutant mice, indicating that per2 plays a role in circadian rhythms of these angiogenic factors.

\section{Significant differences in mRNA levels of angiogenic factors in per2-mutant mice after incision wound injury}

We then designed an experiment to detect the levels of various angiogenic factors after injury as shown in Figure 1.

The per2 mRNA in the wound tissue in control mice showed a pattern of circadian rhythm similar to that in the intact skin (Figure 2). 


\section{Time point selection}

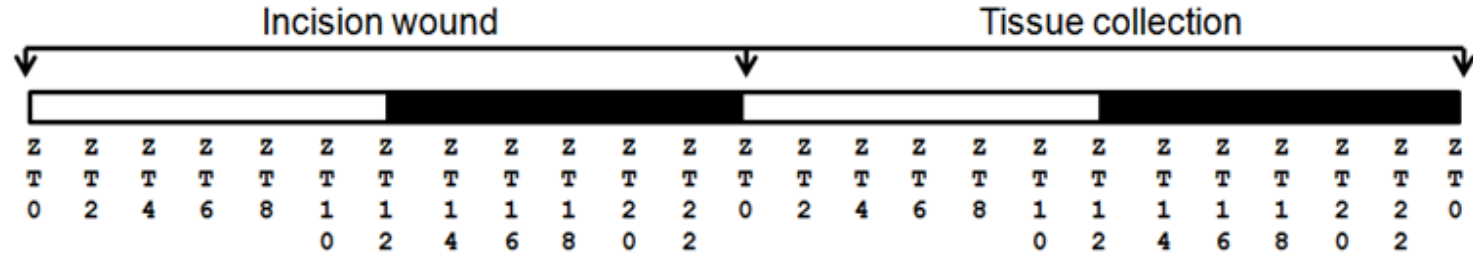

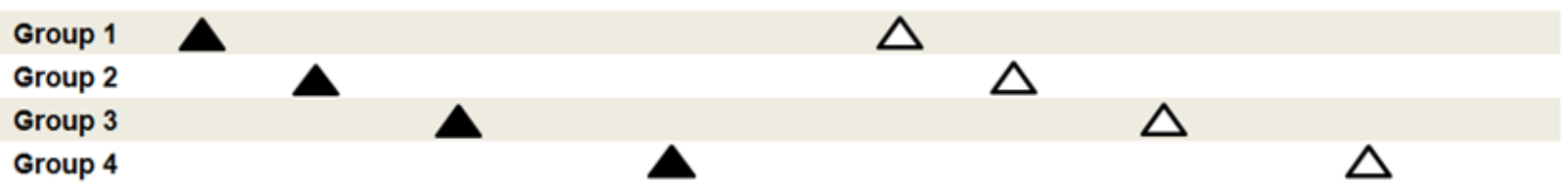

Figure1. Wound experimental design. per2-mutant and control mice were assigned to 4 different groups: 1 (ZT2), 2 (ZT6), 3 (ZT11), and 4 (ZT18). White bars indicate light period, whereas filled bars indicate dark period. Each group had the incision wound performed at a specific ZT time point (filled arrowheads). The skin tissue was collected 24 hours later at the same ZT time point (white arrowheads).

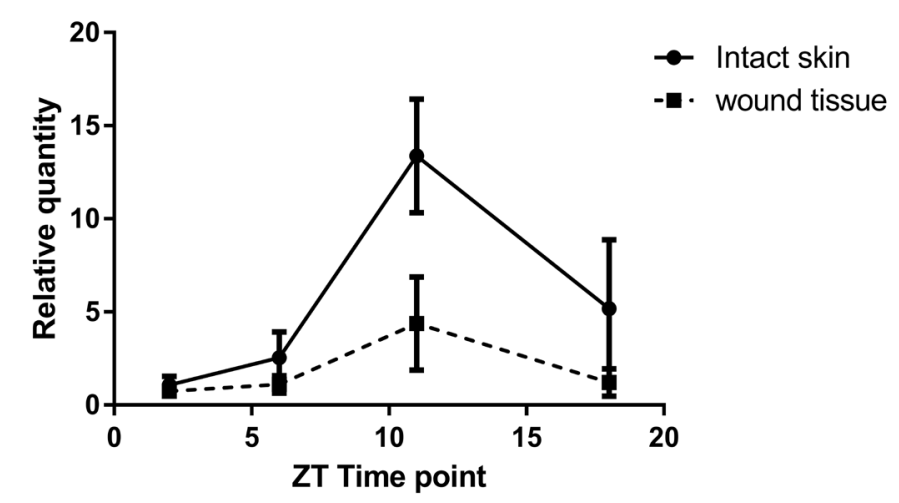

Figure2. The mRNA expressions of per2mRNAinintact skin and wound tissue of control mice at 4 different ZT time points. The solid line refers to intact skin, whereas the broken line refers to wound tissue. The data are expressed as the fold level change from ZT2(relative quantity, $\mathrm{N}=12$ to 14 )

Our comparison of the mRNA expression levels in the wound tissue of per2-mutant mice with those in control mice showed significant differences in the following conditions: at ZT6 for VEGF, at ZT11 for ANGPT2, and at ZT18 for ANGPTL1 (Figure 5). Similar circadian rhythms between the wound tissues of per2-mutant and control mice were observed for CXCL2, MMP9, TGFb1, HGF, and ANGPTL2 (Figure 5). Other angiogenic factor mRNAs showed distinct rhythm patterns between per2-mutant and control mice (Figure 6).

Analysis of the wound tissue among ZT's of per2-mutant mice 24 hours after the skin incision revealed statistically significant differences in mRNA expression levels of VEGF, CXCL2, MMP9, TGFb1, and ANGPTL2, suggesting the existence of per2-independent mechanisms of circadian rhythm for angiogenic factors (Figure 5). FGF1, ANGPT1, CSF3, and PAI1 also showed statistically significant variations among ZT's in per2-mutant mice (Figure 6).

On the other hand, statistically significant differences among ZT's of control mice were detected in mRNA expression levels of CXCL2, MMP9, TGFb1, and ANGPTL2 (Fig 5), and FGF1, ANGPT1, TNMD, and CSF3 (Figure 6).

\section{Angiogenic factor expression levels are independent of the number of leucocytes in wound sites}

We previously showed that after injury, VEGF expression is localized in the leucocytes [3]. We also found that the leucocyte- depleted state by cyclophosphamide resulted in the reduction of VEGF expression [3]. Because leucocyte number could be a factor for fluctuations in angiogenic factor levels, we quantified the leucocytes numbers in samples from wound areas. The histological study showed no statistically significant difference in numbers of leucocytes in the wound areas between per2-mutant and control mice. Neither was there statistical difference between the numbers of leucocytes in the wound areas among ZT's in per2-mutant and in control mice (Figure 7).

Immunohistochemistry analysis of capillaries in the wound area showed no angiogenic difference with per2 deficiency

The above results showed that there are significant differences in expression levels/patterns among mRNAs of the angiogenic factors. These changes could translate into anatomical differences in the healing process after a wound injury. Therefore, we analyzed the vascularization of the wound area using histochemical staining with the endothelial marker CD31. The percentages of CD31-positive vascular areas to the entire wound areas were not statistically significantly different between per2-mutant and control mice 7 days after the skin incision (Figure 8), suggesting that there is no early stage impact as a result of the changes in the angiogenic factor's levels after injury.

\section{Discussion}

Our results show the presence of circadian rhythms of per2 mRNA levels in the intact skin as well as the wound tissue (Figure 2). Our data also show the circadian oscillation of mRNA levels for angiogenic factors in the intact skin of control mice (Figure 3). When compared with control mice, ANGPTL1 and CSF2 mRNAs in the intact skin of per2-mutant mice lose the peak at ZT11 (Figure 4). This indicates the per2-dependency of circadian rhythms of some angiogenic factor mRNA levels. On the other hand, ANGPT2 shows a peak at ZT6 in per2mutant mice (Figure 4). CSF2 and MMP9 mRNAs in the intact skin of per2-mutant mice show increases at ZT2 (Figure 4). These indicate the existence of per2-independent mechanism(s) for circadian rhythms. Circadian rhythms are maintained by several molecular mechanisms [16]. Therefore, our results raise a possibility that per2 disruption in per2-mutant mice may activate other circadian mechanisms, which in turn may influence the circadian change in mRNA expressions. Future studies will be needed to analyze the mechanism of the circadian rhythm of intact skin in per2-mutant mice.

In this study, we have observed that VEGF mRNA relative quantity in the wound was significantly lower at ZT6 in per2-mutant mice 
ZT6 PEAK

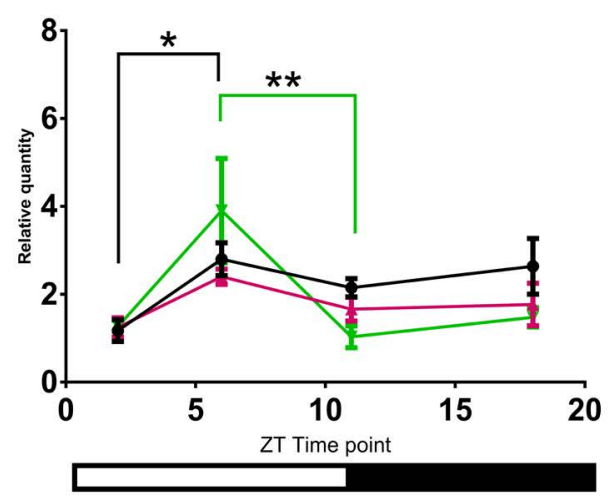

ZT6 - ZT11 PEAK

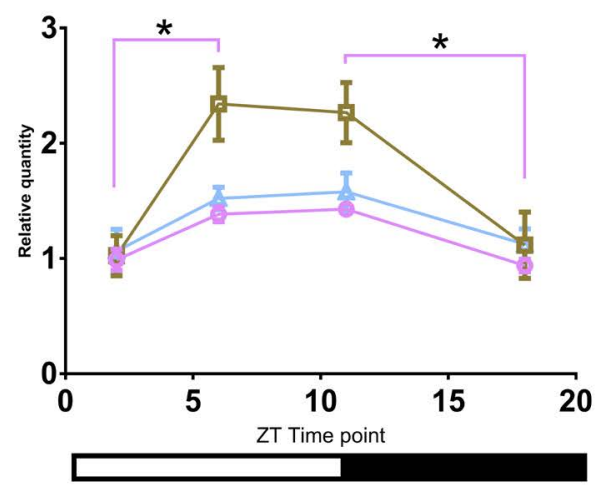

ZT11 PEAK

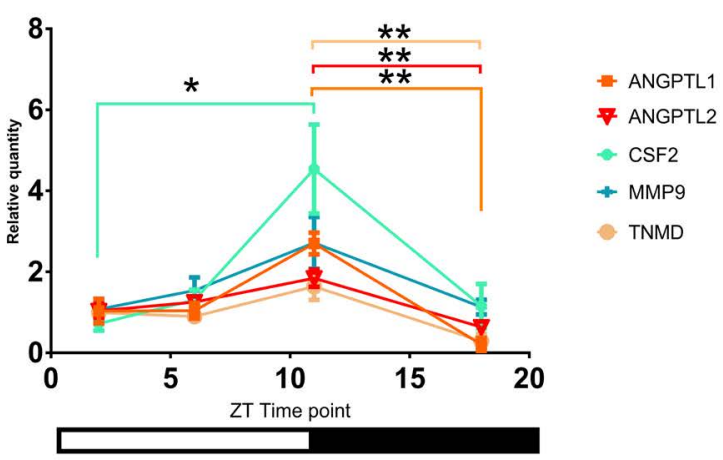

ZT18 PEAK

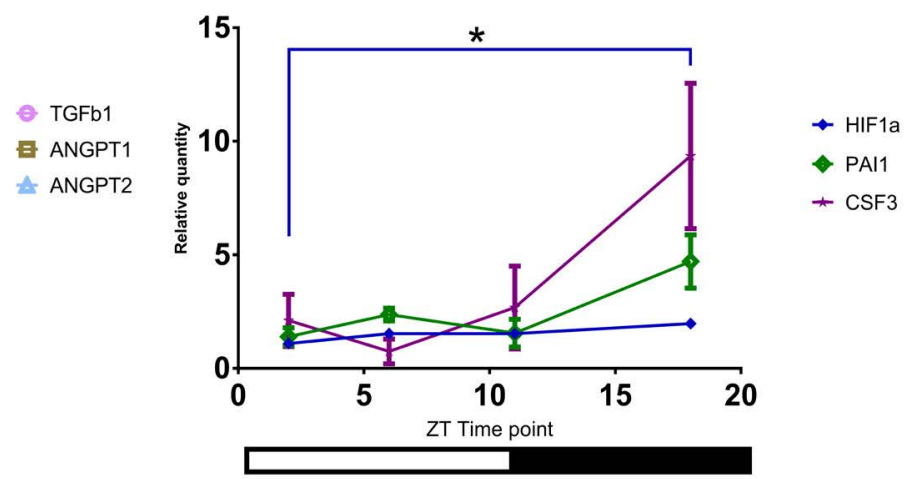

Figure 3. The mRNA levels of different angiogenic factors in control mouse intact skin. Analysis of angiogenic factors' mRNA levels. Of note, four distinct patterns were detected depending on the time of day, as represented by four panels $\left(\mathrm{N}=4\right.$ to 5 ; ${ }^{*} \mathrm{P}<0.05$, $\left.{ }^{* *} \mathrm{P}<0.01\right)$

when compared with control mice (Figure 5). On the other hand, a statistically significant difference between ZT 6 and 18 of per2-mutant mice in the wound was observed for VEGF mRNA expression (Figure 5). Together with the recent findings summarized below, our results point to a view that VEGF mRNA expression by the infiltrating neutrophils in the wound area might be influenced by per2 protein. VEGF has been known to play an important role in the wound healing process. Leucocytes are known to be one of the sources of VEGF [17]. Our previous study showed the presence of VEGF in leucocytes [1]. Cyclophosphamide significantly decreases the numbers of leucocytes in wounds 1 and 3 days after a skin incision [3]. Under this condition, VEGF expression is suppressed, indicating that infiltrating leucocytes in the wound area might be the source of VEGF [3]. Several studies have indicated the circadian VEGF oscillation. VEGF showed a circadian rhythm influenced by circadian clock in human trophoblast cell line [18]. Koyanagi et al. demonstrated that the levels of VEGF mRNA in tumor cells implanted in mice rose substantially in response to hypoxia, but the levels fluctuated rhythmically in a circadian fashion [4]. Per2 in the implanted tumor cells showed a circadian oscillation, and inhibited the hypoxia-induced Vegf promoter activity [4]. Jensen et al. showed that Bmall directly binds to and activates the Vegf promoter via E-boxes [19]. Since it is shown that perl and per2 exhibit circadian oscillations in mRNA expression in mouse peripheral leucocytes [5], it is conceivable that VEGF production in leucocytes could be influenced by per 2 .
In a previous study, per2-mutant mice showed a failure to stabilize HIF1a [10]. Per2 suppressed Clock-Bmal1- and Clock-Bmal2dependent transactivation of the Pail promoter in vitro [11]. A previous study showed that the expression of TGFb1 was dramatically elevated in per2-mutant cholestatic liver [20]. Therefore, we studied other various angiogenic factors in the wound tissue.

Our results from the wound tissue analysis show that ANGPT2 mRNA was statistically significantly higher at ZT11 in per2-mutant mice compared with control mice. ANGPTL1 mRNA expression showed statistically a significantly higher level at ZT18 in per2mutant mice (Figure 5). These are novel findings indicating the per2 involvement in ANGPT2 and ANGPTL1 circadian rhythms.

On the other hand, statistically significant differences among ZT's of per2-mutant mice in the wound were observed for mRNA expressions of VEGF, CXCL2, MMP9, TGFb1, ANGPTL2, FGF1, ANGPT1, CSF3, and PAI1 (Figures 5 and 6), indicating the existence of per2-independent mechanisms of circadian rhythms for angiogenic factors.

Some angiogenic factors including VEGF are produced by leucocytes [1,3]. Our histological study showed no statistically significant fluctuations in leucocyte accumulation in the wound area in per2-mutant and control mice (Figure 7). This indicates that the mRNA oscillations may be due to the intracellular mRNA fluctuations. 


\section{ZT11 peak}

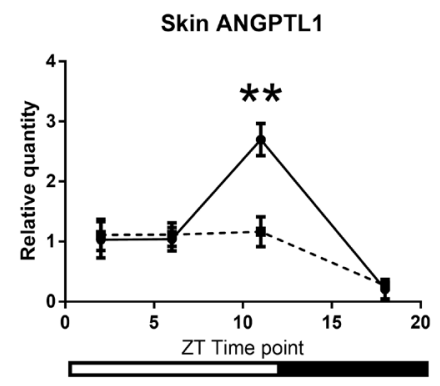

Skin CSF2

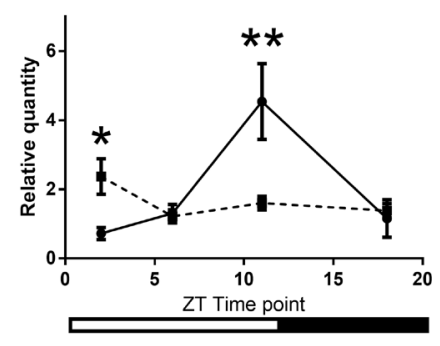

Skin MMP9

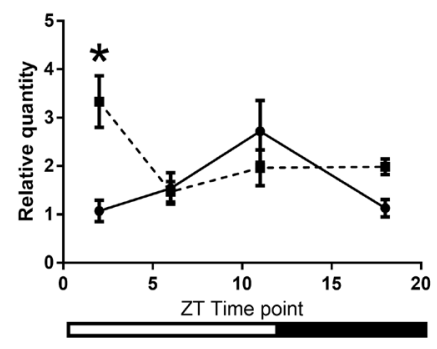

Skin ANGPTL2

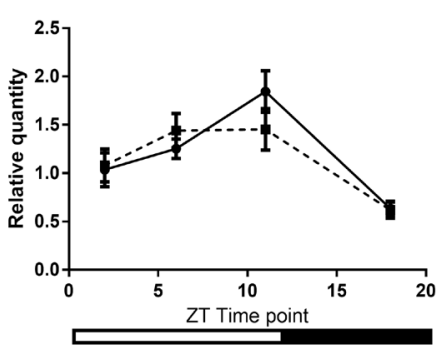

Skin TNMD

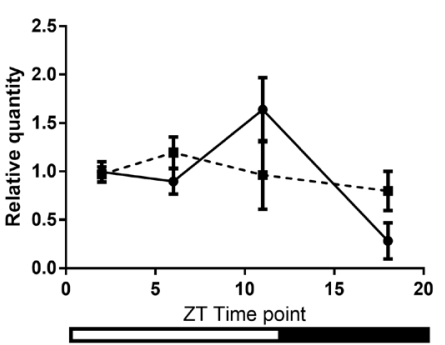

Other angiogenic factors
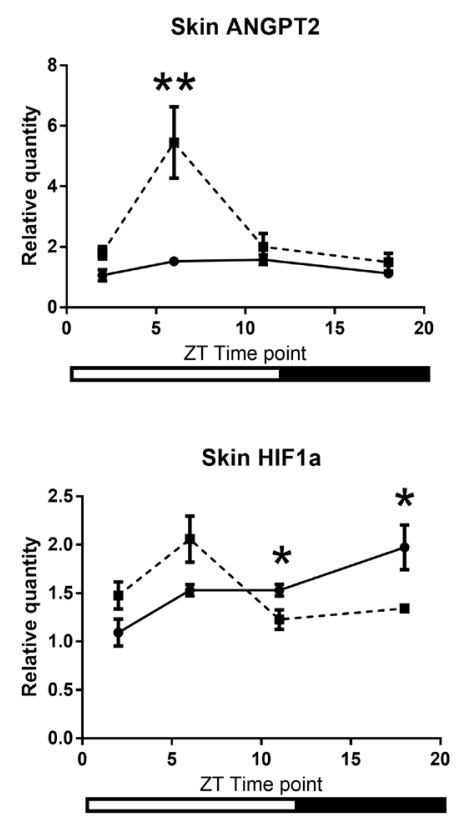

Skin VEGF

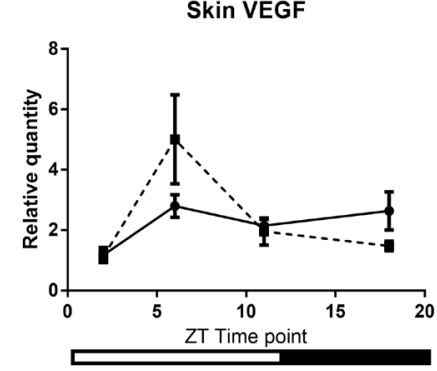

Skin FGF1

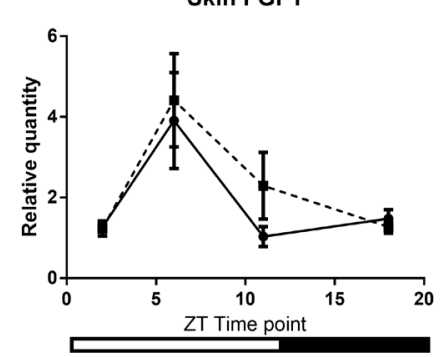

Skin TGFB1a

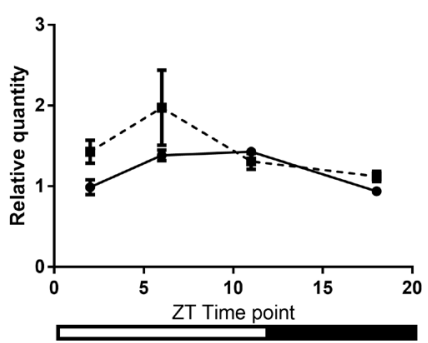

Figure 4. The mRNA levels of different angiogenic factors in per2-mutant mouse skin compared with control mouse skin. Solid lines correspond to control mouse skin and broken lines correspond to per2-mutant mouse skin. Shown is analysis of mRNA from intact skin. per2-mutant mice exhibited distinct circadians patterns for some angiogenic factors relative to control mice. ( $\left.\mathrm{N}=5-9 ;{ }^{*} \mathrm{P}<0.05, * * \mathrm{P}<0.01\right)$. 
VEGF

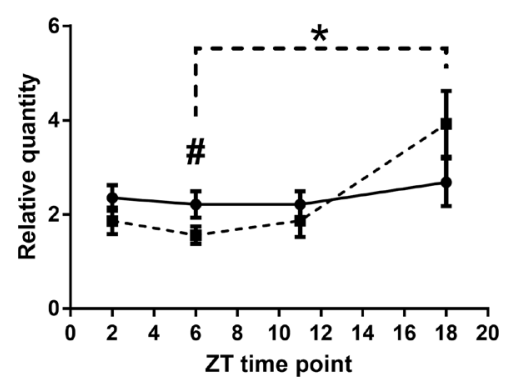

CXCL2

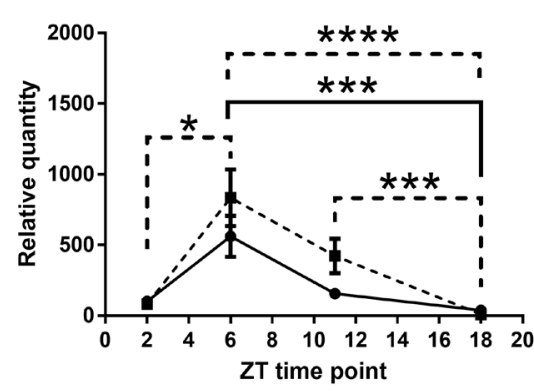

HGF

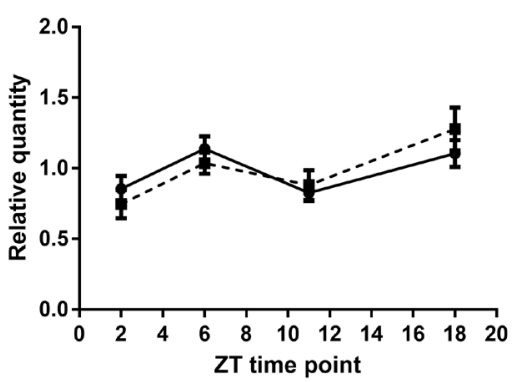

ANGPT2

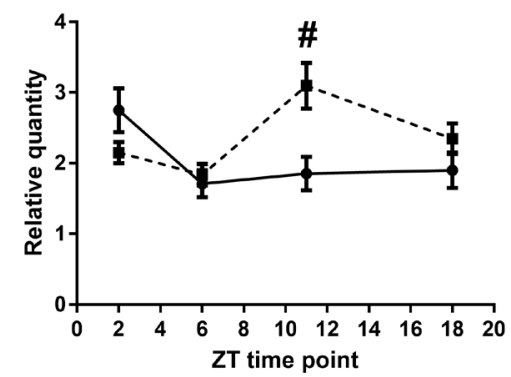

MMP9

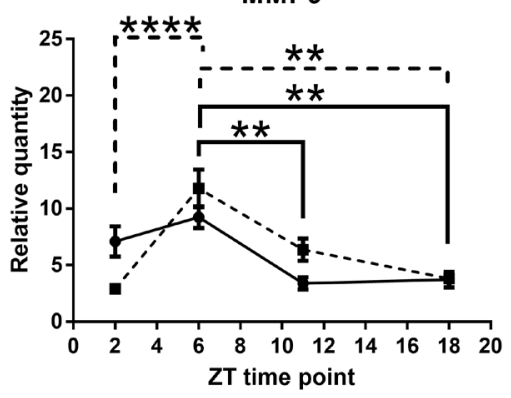

ANGPTL2

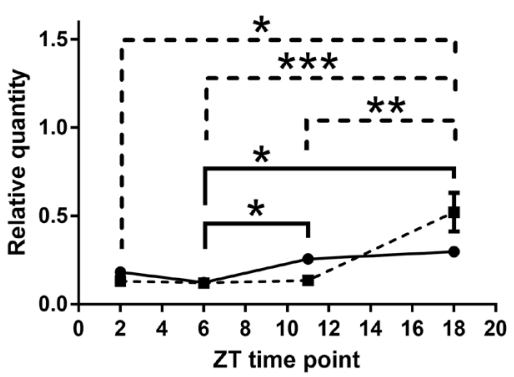

ANGPTL1

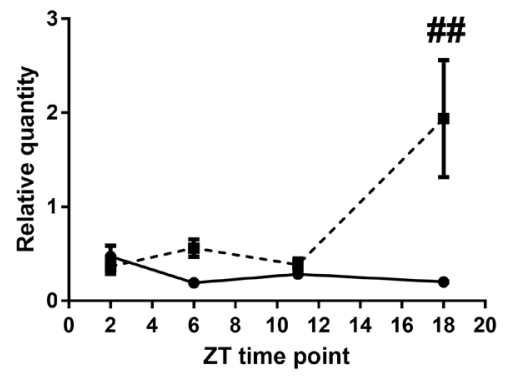

TGFb1

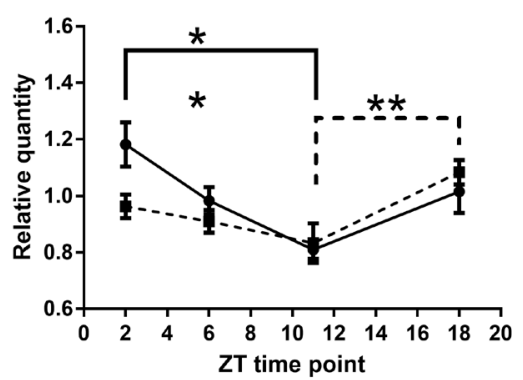

Figure 5. Circadian rhythm patterns of angiogenic factor mRNAs (relative quantity; wound tissues minus intact tissues) of per2-mutant and control mice. Top three panels (VEGF, ANGPT2, and ANGPTL1) show statistically significant differences between per2-mutant and control mice, whereas lower five panels show similar patterns between per2-mutant and control mice. The solid line refers to control mice and the broken lines to per 2 -mutant mice. Number signs (\#) refers to comparison of the same ZT point between control and per2-mutant mice. (N $=10-15 ; * \mathrm{P}<0.05, * * \mathrm{P}<001, * * * \mathrm{P}<0.001, * * * * \mathrm{P}<0.0001 ; \# \mathrm{P}<0.05, \# \# \mathrm{P}<0.01)$

The per2 gene is known to maintain early endothelial progenitor cell function and angiogenesis after myocardial infarction in mice [21]. per2-mutant mice showed increased vascular senescence and impaired endothelial progenitor cell function [22]. Therefore, we used immunohistochemistry to analyze the CD31-positive endothelial cells in the wound area. CD31-positve vessel areas were not statistically significantly different between per2-mutant and control mice in the wound tissue 7 days after the skin incision. Therefore, the angiogenic response to the wounds in histology assessed after 7 days was not different between per2-mutant and control mice (Figure 8). Kowalska et al. demonstrated that circadian clock-deficient per $1 /$ per $2^{\text {mut }}$ and Bmal1 /- mice showed defective wound healing [23]. Additional research is needed to understand thoroughly the effects of per 2 deficiency in wound healing process.

\section{Conclusions}

Our study demonstrates a novel finding that angiogenic factor mRNA expressions in the mouse skin wound are under the influence of per2 gene. Our results also indicate the per2-independent mechanism of circadian rhythms for some angiogenic factor mRNAs. Additional research will be needed to understand more clearly how circadian rhythm influences the healing process.

\section{Acknowledgements}

This study was supported by JSPS KAKENHI Grant number $15 \mathrm{~K} 08883$. 


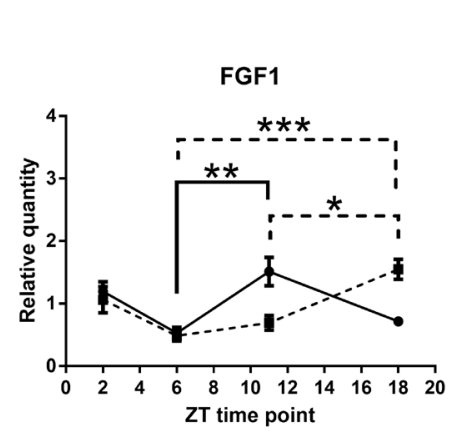

HIF1a

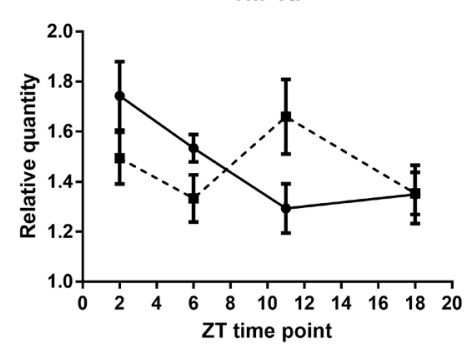

PAl1

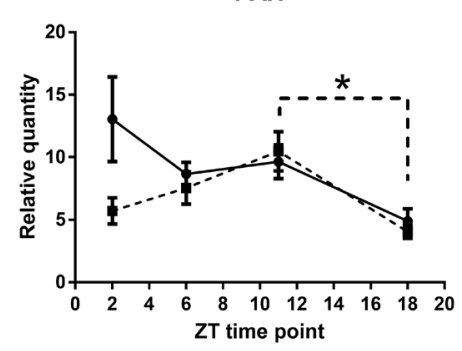

ANGPT1

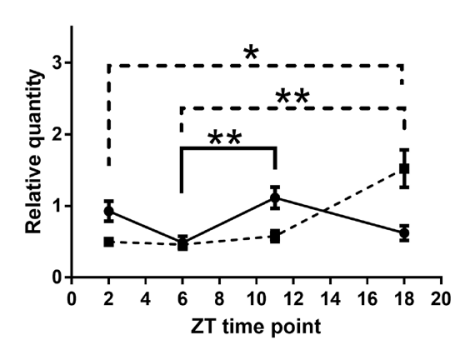

CSF2

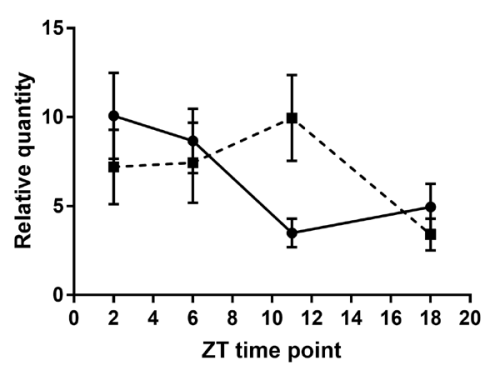

TNMD

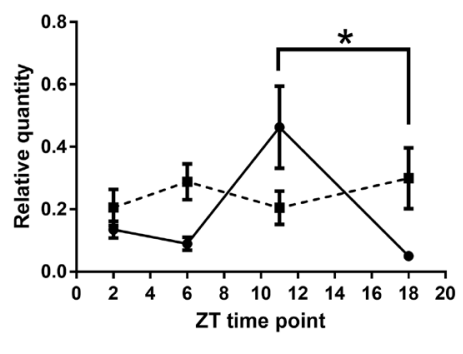

Figure 6. Other Various circadian rhythm patterns of angiogenic factor mRNAs (relative quantity; wound tissues minus intact tissues) of per2-mutant and control mice. The solid line refers to control mice and the broken line to per2-mutant mice. $(\mathrm{N}=9-15 ; * \mathrm{P}<0.05, * * \mathrm{P}<001, * * * \mathrm{P}<0.001)$.

A

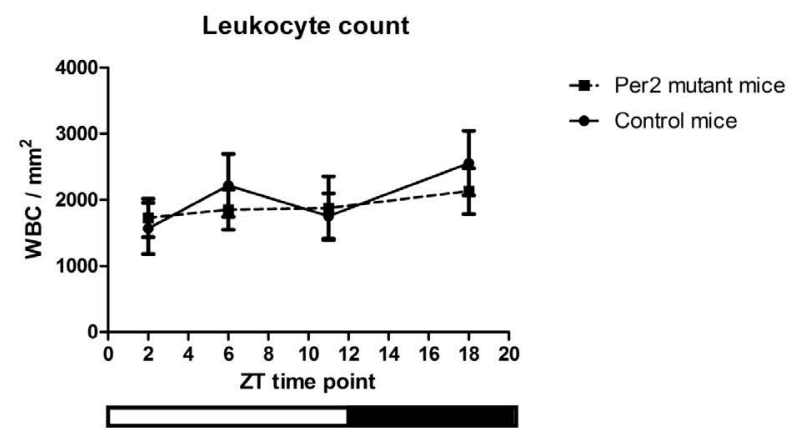

B

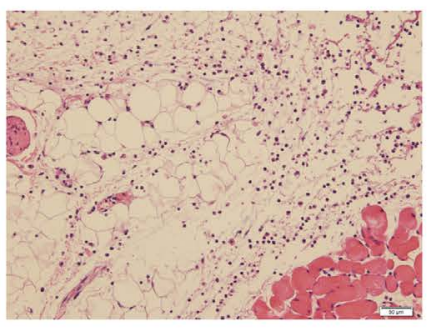

Per2 mutant mice

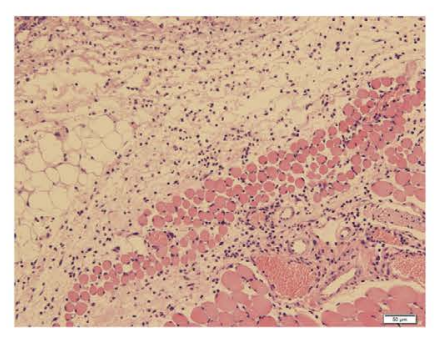

Control mice

Figure 7. Leucocyte quantification in skin wound samples. A. The numbers of leucocytes in the wound areas of per2-mutant and control mice ( $\mathrm{N}=7$ - 13 samples for each group). B Representative pictures of HE-stained wound tissues from control and per2-mutant mice. Scale bars show 100 micrometers. 

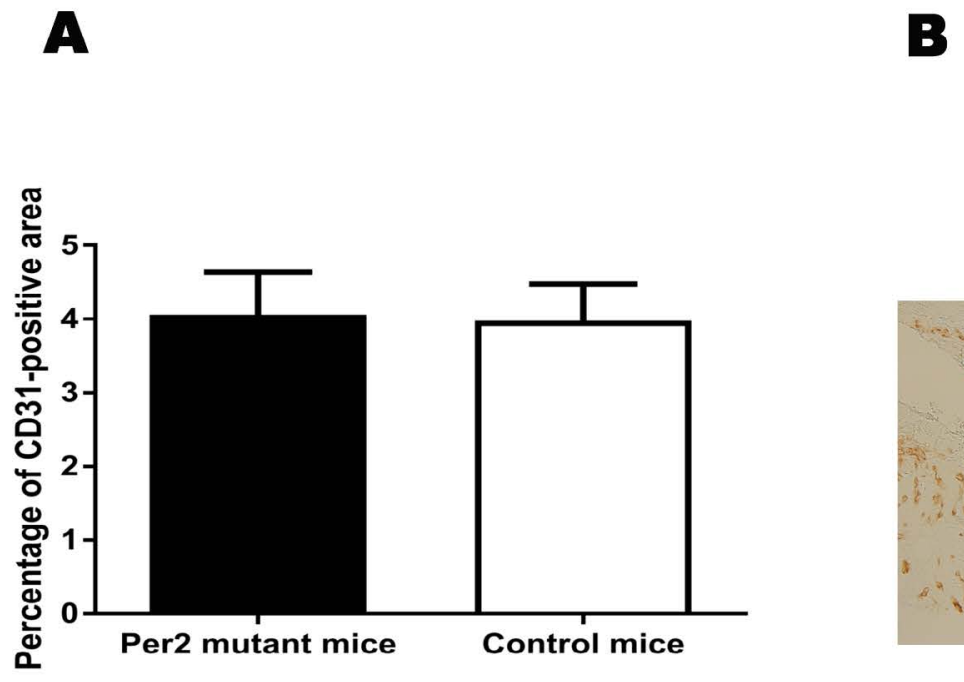

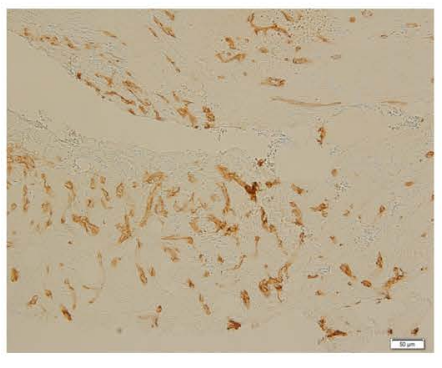

Per2 mutant mice

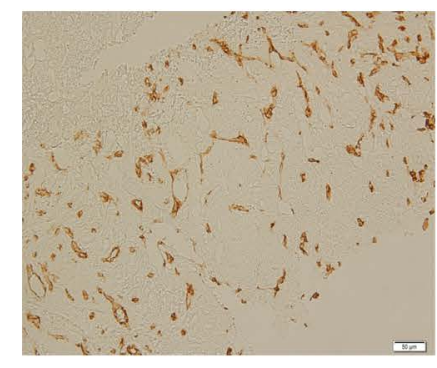

Control mice

Figure 8. The evaluation of CD31-positive vascular areas in the wound areas in per2-mutant and control mice.A. The percentages of CD31-positive vascular areas in the wound areas. $\mathrm{N}=9$ mice per group. B. Representative pictures of CD31-stained tissues from per2-mutant and control mice.

\section{References}

1. Nogami M, Hoshi T, Kinoshita M, Arai T, Takama M, et al. (2007) Vascular endothelial growth factor expression in rat skin incision wound. Med Mol Morphol 40: 82-87. [Crossref]

2. Nogami M, Hoshi T, Arai T, Toukairin Y, Takama M, et al. (2009) Morphology of lymphatic regeneration in rat incision wound healing in comparison with vascular regeneration. Leg Med (Tokyo) 11: 213-218. [Crossref]

3. Nogami M, Hoshi T, Toukairin Y, Arai T (2010) Vascular endothelial growth factor in the early stage of skin incision wounds in cyclophosphamide-induced leukocytopenic rats. Leg Med (Tokyo) 12: 128-131. [Crossref]

4. Koyanagi S, Kuramoto Y, Nakagawa H, Aramaki H, Ohdo S, et al. (2003) A molecular mechanism regulating circadian expression of vascular endothelial growth factor in tumor cells. Cancer Res 63: 7277-7283. [Crossref]

5. Fukuya H, Emoto N, Nonaka H, Yagita K, Okamura H, et al. (2007) Circadian expression of clock genes in human peripheral leukocytes. Biochem Biophys Res Commun 354: 924-928. [Crossref]

6. Takata M, Burioka N, Ohdo S, Takane H, Terazono H, et al. (2002) Daily expression of mRNAs for the mammalian Clock genes Per2 and clock in mouse suprachiasmatic nuclei and liver and human peripheral blood mononuclear cells. Jpn J Pharmacol 90: 263-269. [Crossref]

7. James FO, Boivin DB, Charbonneau S, Belanger V, Cermakian N (2007) Expression of clock genes in human peripheral blood mononuclear cells throughout the sleep/wake and circadian cycles. Chronobiol int 24: 1009-1034. [Crossref]

8. Kusanagi H, Hida A, Satoh K, Echizenya M, Shimizu T, et al. (2008) Expression profiles of 10 circadian clock genes in human peripheral blood mononuclear cells. Neurosci Res 61: 136-142. [Crossref]

9. Virag JA, Dries JL, Easton PR, Friesland AM, DeAntonio JH, et al. (2010) Attenuation of myocardial injury in mice with functional deletion of the circadian rhythm gene mPer2. Am J Physiol Heart Circ Physiol 298: H1088-1095. [Crossref]

10. Eckle T, Hartmann K, Bonney S, Reithel S, Mittelbronn M, et al. (2012) Adora2belicited Per2 stabilization promotes a HIF-dependent metabolic switch crucial for myocardial adaptation to ischemia. Nat Med 18: 774-782. [Crossref]

11. Oishi K, Miyazaki K, Uchida D, Ohkura N, Wakabayashi M, et al. (2009) PERIOD2 is a circadian negative regulator of PAI-1 gene expression in mice. J Mol Cell Cardiol 46: 545-552. [Crossref]
12. Zheng B, Larkin DW, Albrecht U, Sun ZS, Sage M, et al. (1999) The mPer2 gene encodes a functional component of the mammalian circadian clock. Nature 400: 169173. [Crossref]

13. Kameyama H, Udagawa O, Hoshi T, Toukairin Y, Arai T, et al. (2015) The mRNA expressions and immunohistochemistry of factors involved in angiogenesis and lymphangiogenesis in the early stage of rat skin incision wounds. Leg Med (Tokyo) 17: 255-260. [Crossref]

14. Livak KJ, Schmittgen TD (2001) Analysis of relative gene expression data using realtime quantitative PCR and the 2(-Delta Delta C(T)) Method. Methods 25: 402-408. [Crossref]

15. Tanioka M, Yamada H, Doi M, Bando H, Yamaguchi Y, et al. (2009) Molecular clocks in mouse skin. J Invest Dermatol 129: 1225-1231. [Crossref]

16. Siepka SM, Yoo SH, Park J, Lee C, Takahashi JS (2007) Genetics and neurobiology of circadian clocks in mammals. Cold Spring Harb Symp Quant Biol 72: 251-259. [Crossref]

17. Lin YJ, Lai MD, Lei HY, Wing LY (2006) Neutrophils and macrophages promote angiogenesis in the early stage of endometriosis in a mouse model. Endocrinology 147: 1278-1286. [Crossref]

18. Frigato E, Lunghi L, Ferretti ME, Biondi C, Bertolucci C (2009) Evidence for circadian rhythms in human trophoblast cell line that persist in hypoxia. Biochem Biophys Res Commun 378: 108-111. [Crossref]

19. Jensen LD, Cao Z, Nakamura M, Yang Y, Brautigam L, et al. (2012) Opposing effects of circadian clock genes bmal1 and period2 in regulation of VEGF-dependent angiogenesis in developing zebrafish. Cell Rep 2: 231-241. [Crossref]

20. Chen P, Kakan X, Wang S, Dong W, Jia A, et al. (2013) Deletion of clock gene Per2 exacerbates cholestatic liver injury and fibrosis in mice. Exp Toxicol Pathol 65: 427 432. [Crossref]

21. Sun YY, Bai WW, Wang B, Lu XT, Xing YF, et al. (2014) Period 2 is essential to maintain early endothelial progenitor cell function in vitro and angiogenesis after myocardial infarction in mice. J Cell Mol Med 18: 907-918. [Crossref]

22. Wang CY, Wen MS, Wang HW, Hsieh IC, Li Y, et al. (2008) Increased Vascular Senescence and Impaired Endothelial Progenitor Cell Function Mediated by Mutation of Circadian Gene Per2. Circulation 118: 2166-2173. [Crossref]

23. Kowalska E, Ripperger JA, Hoegger DC, Bruegger P, Buch T, et al. (2013) NONO couples the circadian clock to the cell cycle. Proc Natl Acad Sci U S A 110: 1592-1599. [Crossref]

Copyright: $@ 2017$ Hoshi T. This is an open-access article distributed under the terms of the Creative Commons Attribution License, which permits unrestricted use, distribution, and reproduction in any medium, provided the original author and source are credited. 